\title{
Invertibles in topological rings: a new approach
}

\author{
Francisco Javier García-Pacheco ${ }^{1} \cdot$ Alejandro Miralles ${ }^{2} \cdot$ Marina Murillo-Arcila $^{3}$
}

Received: 29 March 2021 / Accepted: 26 October 2021 / Published online: 15 November 2021

(c) The Author(s) under exclusive licence to The Royal Academy of Sciences, Madrid 2021

\begin{abstract}
Every element in the boundary of the group of invertibles of a Banach algebra is a topological zero divisor. We extend this result to the scope of topological rings. In particular, we define a new class of semi-normed rings, called almost absolutely semi-normed rings, which strictly includes the class of absolutely semi-valued rings, and prove that every element in the boundary of the group of invertibles of a complete almost absolutely semi-normed ring is a topological zero divisor. To achieve all these, we have to previously entail an exhaustive study of topological divisors of zero in topological rings. In addition, it is also well known that the group of invertibles is open and the inversion map is continuous and $\mathbb{C}$-differentiable in a Banach algebra. We also extend these results to the setting of complete normed rings. Finally, this study allows us to generalize the point, continuous and residual spectra to the scope of Banach algebras.
\end{abstract}

Keywords Spectrum · Rings · Algebras · Zero divisor $\cdot$ Invertibles $\cdot$ Operator

Mathematics Subject Classification $16 \mathrm{~W} 80 \cdot 47 \mathrm{~L} 10 \cdot 47 \mathrm{~A} 10$

\section{Introduction}

In this manuscript we generalize the point, continuous and residual spectra of an operator to algebras. We will also analyse the topological properties of the group of invertibles [1-3] and the topological divisors of zero [4-6] in general topological rings. We also refer the reader to [7-9] for further information about extending the classical Operator Spectral Theory to

$\triangle$ Marina Murillo-Arcila

mamuar1@upv.es

Francisco Javier García-Pacheco

garcia.pacheco@uca.es

Alejandro Miralles

mirallea@uji.es

1 Departamento de Matemáticas, Escuela Superior de Ingeniería, Universidad de Cádiz, 11510 Puerto Real, Spain

2 IMAC and Departament de Matemàtiques, Universitat Jaume I, 12071 Castelló de la Plana, Spain

3 Instituto Universitario de Matemática Pura y Aplicada, Universitat Politècnica de València, 46022 València, Spain 
the scope of Banach algebras through the Gelfand Theory and the Continuous Functional Calculus. See also the excellent books and monographs [8,10-16].

The paper is organized as follows: Sect. 2 gathers all the necessary background we will need. Section 3 is divided into three subsections: in the first one, Sect. 3.1, we introduce the novel concept of completely uniform algebra and we prove that $C(K)$, the algebra of continuous functions on a compact space $K$, is completely uniform. We also characterize the topological divisors of zero of completely uniform algebras. Section 3.2 deals with the spectra of Banach algebras and we extend the notions of point, continuous and residual spectra to the scope of Banach algebras. Furthermore, we prove that the spectral decomposition in the algebra of bounded operators also holds for general Banach algebras. As an illustrative example, we characterize these spectra for $C(K)$. We also compute the approximated spectra of completely uniform algebras. Finally, in Sect. 3.3 we accomplish the global differentiability of the inversion map in topological rings. We introduce two concepts: absolutely invertibles and absolutely semi-normed rings. We prove that the boundary of the group of invertibles in complete absolutely normed rings is contained in the set of bilateral topological divisors of zero. We also illustrate nontrivial examples of absolutely normed rings which are not absolutely valued. These new concepts are also interpreted in the algebra of bounded operators and $C(K)$.

\section{Preliminaries}

Throughout the sequel, all the rings will be considered associative and unitary. All algebras will be considered over the reals except for $*$-algebras and uniform algebras, which will be considered over the complex numbers. Whenever we talk about the spectrum of an element of an algebra, such algebra will also be considered over the complex field. In this section we compile some definitions and results that will be needed throughout this manuscript.

\subsection{Topological background}

If $X$ is a topological space and $A$ is a subset of $X$, then $\operatorname{int}(A), \operatorname{cl}(A), \operatorname{bd}(A)$ stand for the interior, the closure and the boundary of $A$, respectively. If $x \in X$, then by $\mathcal{N}_{x}(X)$ we intend to denote the filter of neighborhoods of $x$. If there is no confusion with $X$, then we will simply denote it by $\mathcal{N}_{x}$.

The classical characterization of module topology [17, Theorem 3.6] will be very much employed throughout this manuscript. We refer the reader to $[17,18]$ for a wider perspective on topological rings and modules. In [19, Theorem (1)], it was proved that, for any topological module $M, \bigcap \mathcal{N}_{0}(M):=\bigcap_{V \in \mathcal{N}_{0}(M)} V$ is a closed submodule of $M$ whose inherited topology is the trivial topology.

A $*$-ring is a ring $R$ endowed with an additive, anti-multiplicative, involution $*: R \rightarrow R$. It easily follows that $0^{*}=0$ and $1^{*}=1$, in fact, $\left(u^{-1}\right)^{*}=\left(u^{*}\right)^{-1}$ for every $u \in \mathcal{U}(R)$, where $\mathcal{U}(R)$ denotes the set of invertibles of $R$. If $C \subset R$, we will denote $C^{*}:=\left\{r^{*}: r \in C\right\}$. A $*$-algebra is an algebra which is a $*$-ring whose involution is conjugate linear. A topological $*$-ring is a topological ring which is endowed with a continuous involution. 


\subsection{Semi-norms and absolute semi-values}

A semi-normed group is a pair $(G,\|\cdot\|)$ where $(G,+)$ is an additive group and $\|\cdot\|: G \rightarrow$ $[0, \infty)$ is a function satisfying the following properties:

- Identity preserving: $\|0\|=0$.

- Symmetricity: $\|-g\|=\|g\|$ for any $g \in G$.

- Triangular inequality: $\|f+g\| \leq\|f\|+\|g\|$ for any $f, g \in G$.

If, in addition, $\|g\|=0$ implies $g=0$, then the semi-norm is called a norm. If $\|f+g-f\|=$ $\|g\|$ for all $f, g \in G$, the semi-norm is called conjugation-invariant. Notable subsets of semi-normed groups are the closed unit ball $\mathrm{B}_{G}:=\{g \in G:\|g\| \leq 1\}$, the open unit ball $\mathrm{U}_{G}:=\{g \in G:\|g\|<1\}$ and the unit sphere $\mathrm{S}_{G}:=\{g \in G:\|g\|=1\}$.

An absolute semi-value (value) on a ring $R$ is a group semi-norm (norm) $|\cdot|$ on the additive group of $R$ which is also multiplicative, that is, $|r s|=|r||s|$ for all $r, s \in R$. Either $|1|=1$ or $|1|=0$. If $|1|=1$, then $\left|r^{-1}\right|=|r|^{-1}$ for all $r \in \mathcal{U}(R)$, where $\mathcal{U}(R)$ denotes the multiplicative group of invertibles of $R$. If $|1|=0$, then $|\cdot|=0$.

A semi-norm (norm) on a ring $R$ is a group semi-norm (norm) $\|\cdot\|$ on the additive group of $R$ which is also submultiplicative, that is, $\|r s\| \leq\|r\|\|s\|$ for all $r, s \in R$. It satisfies that either $\|1\|=0$ or $\|1\| \geq 1$. If $\|1\|=1$, then we call it a unital ring semi-norm. In every semi-normed ring $R,\|a\|\left\|b^{-1}\right\|^{-1} \leq\|a b\| \leq\|a\|\|b\|$ for all $a \in R$ and $b \in \mathcal{U}(R)$. Absolutely semi-values are examples of ring semi-norms.

\subsection{Divisors of zero}

Let $R$ be a ring and $s \in R$. We will denote by $\ell d(s)$ the set of left divisors of $s$ which is given by:

$$
\ell d(s):=\{r \in R: \text { there exists } t \in R \backslash\{0\} \text { with } r t=s\} .
$$

Similarly, $r d(s)$ stands for the set of right divisors of $s$. Recall that $R$ is called an integral domain if $\ell d(0)=r d(0)=\{0\}$. Notice that $\ell d(1)(r d(1))$ is precisely the set of elements admitting a right-(left-)inverse. Because of the associativity of $R$, it is clear that $\mathcal{U}(R)=\ell d(1) \cap r d(1)$. Observe also that $\ell d(0) \cap r d(1)=\varnothing=r d(0) \cap \ell d(1)$. In particular, $(\ell d(0) \cup r d(0)) \cap \mathcal{U}(R)=\varnothing$. In general we have that $\ell d(0) \cap \ell d(1) \neq \varnothing$ and $r d(0) \cap r d(1) \neq \varnothing$.

Example 1 Let $X$ be an infinite dimensional vector space. Consider two nonzero vector subspaces $U, V$ of $X$ such that $X=U \oplus V$ and $\operatorname{dim}(U)=\operatorname{dim}(X)$. Let $T: U \rightarrow X$ be an isomorphism and let $P: X \rightarrow X$ the projection on $U$. Notice that $T \circ P$ is a right divisor of zero of $\mathcal{L}(X)$ since $(T \circ P) \circ(I-P)=0$. Also, $T \circ P$ is right-invertible in $\mathcal{L}(X)$, that is, it is a right divisor of $I$ since $(T \circ P) \circ T^{-1}=I$, where $T^{-1}: X \rightarrow U$ is the inverse map of $T$. Hence, $T \circ P \in \operatorname{rd}(0) \cap \operatorname{rd}(I)$.

The following result [20, Theorem 57.4] characterizes the divisors of zero in the algebra $\mathcal{B}(X)$ of continuous linear operators on a normed space $X$.

Theorem 1 Let $X$ be a normed space. Then:

$$
\ell d(0)=\{T \in \mathcal{B}(X): \operatorname{ker}(T) \neq\{0\}\} \text { and } r d(0)=\{T \in \mathcal{B}(X): \overline{T(X)} \subsetneq X\} .
$$


It is clear that $\ell d(r)^{*}=r d\left(r^{*}\right)$ and $r d(r)^{*}=\ell d\left(r^{*}\right)$ in every $*$-ring. Notice that $\mathcal{B}(X)$ is not in general a $*$-ring unless $X$ is a Hilbert space. For linear operators $T: X \rightarrow X$ we can state that $\ell d(T)^{*} \subseteq r d\left(T^{*}\right)$ and $r d(T)^{*} \subseteq \ell d\left(T^{*}\right)$. The equalities hold if $X$ is reflexive.

Remark 1 It is well known [21, Lemma 3.1.16] that a continuous linear operator $T: X \rightarrow X$ on a normed space $X$ satisfies that $\operatorname{ker}\left(T^{*}\right)=T(X)^{\perp}$. Therefore, $T$ has dense range if and only if $\operatorname{ker}\left(T^{*}\right)=\{0\}$, where $T^{*}: X^{*} \rightarrow X^{*}$ denotes the dual operator of $T$. In other words, according to Example 1, $T \in \operatorname{rd}(0)$ if and only if $T^{*} \in \ell d(0)$ even if $X$ is not reflexive.

\subsection{Topological divisors of zero}

Let $R$ be a topological ring. An element $r \in R$ is said to be a topological left divisor of 0 provided that there exists a subset $S \subseteq R$ such that $0 \notin \operatorname{cl}(S)$ and $0 \in \operatorname{cl}(r S)$. The set of topological left divisors of 0 is denoted by $t \ell d(0)$. In a similar way, the topological right divisors of 0 can be defined and they are denoted by $\operatorname{trd}(0)$. The following proposition, whose proof we omit, highlights the basic properties of topological divisors of zero.

Proposition 1 Let $R$ be a topological ring. Then:

1. $r d(1) \cap t \ell d(0)=\varnothing=\ell d(1) \cap \operatorname{trd}(0)$. In particular, $(t \ell d(0) \cup \operatorname{trd}(0)) \cap \mathcal{U}(R)=\varnothing$.

2. If the ring topology of $R$ is trivial, then $t \ell d(0)=\operatorname{trd}(0)=\varnothing$.

3. If the ring topology of $R$ is not trivial, then $\bigcap \mathcal{N}_{0}(R) \subseteq \operatorname{t\ell d}(0) \cap \operatorname{trd}(0)$.

4. If $R$ is Hausdorff, then $\ell d(0) \subseteq t \ell d(0)$ and $r d(0) \subseteq \operatorname{trd}(0)$.

5. If $R$ is discrete, then $\ell d(0)=t \ell d(0)$ and $r d(0)=\operatorname{trd}(0)$.

Let $R$ be a semi-normed ring. Denote $\tau_{R}:=\sup \{\|r\|: r \in R\}$. For every $0<\varepsilon<\tau_{R}$, we define the left- $\varepsilon$-minimum norm of an element $r \in R$ as $\|r\|_{\ell \varepsilon}:=\inf \{\|r s\|:\|s\| \geq \varepsilon\}$. The right- $\varepsilon$-minimum norm is defined analogously. Notice that if $0<\delta<\varepsilon$, then $\|r\|_{\ell \delta} \leq\|r\|_{\ell \varepsilon}$. The following proposition is an easy characterization of topological left divisors of zero, the details of whose proof we spare to the reader. Observe that a similar characterization can be provided for topological right divisors of zero.

Proposition 2 Let $R$ be a seminormed ring. The following conditions are equivalent for an element $r \in R$ :

1. $r \in t \ell d(0)$.

2. $\|r\|_{\ell \varepsilon}=0$ for some $0<\varepsilon<\tau_{R}$.

3. There exists a sequence $\left(s_{n}\right)_{n \in \mathbb{N}} R$ such that $\inf _{n \in \mathbb{N}}\left\|s_{n}\right\|>0$ and $\left(r s_{n}\right)_{n \in \mathbb{N}}$ converges to 0 .

In case $R$ is a semi-normed algebra and $r \in R$, then $\tau_{R}=\infty$ and

$$
\|r\|_{\ell \varepsilon}=\inf \{\|r s\|:\|s\|=\varepsilon\}=\varepsilon\|r\|_{\ell 1} .
$$

Also, $r \in t \ell d(0)$ if and only if $\|r\|_{\ell 1}=0$, that is, if and only if there exists a sequence $\left(s_{n}\right)_{n \in \mathbb{N}} \subseteq \mathrm{S}_{R}$ so that $\left(r s_{n}\right)_{n \in \mathbb{N}}$ converges to 0 .

Note that if $R$ is a semi-normed ring whose unit sphere is compact, then $t \ell d(0) \subseteq \ell d(0)$. As a consequence, if $A$ is a finite dimensional normed algebra, then $\ell d(0)=t \ell d(0)$.

The following theorem stated in [20, Section 57] characterizes the set of topological left divisors of zero which are not left divisors of zero in $\mathcal{B}(X)$. In what follows, given $T \in \mathcal{B}(X)$, the minimum-norm of $T$ is defined as $\|T\|_{\min }:=\inf \left\{\|T(x)\|: x \in \mathrm{S}_{X}\right\}$. 
Theorem 2 Let $X$ be a normed space and $T \in \mathcal{B}(X)$. Then $T \in t \ell d(0)$ in $\mathcal{B}(X)$ if and only if $\|T\|_{\min }=0$. On the other hand, if $X$ is complete, then:

$$
t \ell d(0) \backslash \ell d(0)=\{T \in \mathcal{B}(X): \operatorname{ker}(T)=\{0\} \text { and } T(X) \text { is not closed }\} .
$$

Similar to the divisors of zero, it is clear that $t \ell d(0)^{*}=\operatorname{trd}(0)$ and $\operatorname{trd}(0)^{*}=t \ell d(0)$ in every $*$-ring. As we have mentioned, $\mathcal{B}(X)$ is not in general a $*$-ring. We have that $t \ell d(0)^{*} \subseteq \operatorname{trd}(0)$ and $\operatorname{trd}(0)^{*} \subseteq t \ell d(0)$ in $\mathcal{B}(X)$. The equalities hold if $X$ is reflexive.

The following proposition, whose proof we omit, is a version of Remark 1 for topological divisors of zero:

Proposition 3 Let $X$ be a normed space and $T \in \mathcal{B}(X)$. Then $T \in \operatorname{trd}(0)$ if and only if $T^{*} \in t \ell d(0)$.

\subsection{Uniform algebras}

If we deal with a Banach algebra $(A,\|\cdot\|)$, its spectrum is given by:

$$
M_{A}:=\{\phi: A \rightarrow \mathbb{C}: \text { homomorphisms of algebras such that } \phi \neq 0\}
$$

which is a subset of the unit sphere of $A^{*}$ and it is a compact space endowed with the $w^{*}$ topology (see [12]). For any $a \in A$ we define $\|a\|_{M_{A}}:=\sup _{\phi \in M_{A}}|\phi(a)|$ and it is clear that $\|a\|_{M_{A}} \leq\|a\|$. We can also define the so-called minimum norm associated to $M_{A}$ as follows:

$$
\|a\|_{M_{A} \min }:=\inf _{\phi \in M_{A}}|\phi(a)| .
$$

A Banach algebra $A$ is called a uniform algebra if $\|\cdot\|_{M_{A}}=\|\cdot\|$. If $A$ is commutative, then this is equivalent to being isometrically isomorphic to a closed Banach subalgebra of a $C(K)$ space which separates points of $K$ and contains the constant function 1.

\section{Main results}

\subsection{Completely uniform algebras}

Given a Banach algebra $A$ with spectrum $M_{A}, a \in A$ and $\phi \in M_{A}$, we define $\phi(a \bullet) \in A^{*}$ as $\phi(a \bullet)(b)=\phi(a b)$ for all $b \in A$. It is clear that $\|\phi(a \bullet)\| \leq|\phi(a)|$ and $\|\phi(a \bullet)\|=|\phi(a)|$.

Definition 1 A uniform algebra $A$ is called completely uniform if for every $a \in A$ and $\phi \in M_{A}$, there exists a sequence $\left(b_{n}\right)_{n \in \mathbb{N}} \subseteq \mathrm{S}_{A}$ such that $\left\|a b_{n}\right\|-\left|\phi\left(a b_{n}\right)\right| \rightarrow 0$ as $n \rightarrow \infty$.

Theorem 3 Let $K$ be a Hausdorff compact space. Then $C(K)$ is a completely uniformalgebra.

Proof It is well-known that $M_{C(K)}=\left\{\delta_{x}: x \in K\right\}$. Hence it only suffices to show that for all $f \in C(K)$ and all $x \in K$ there exists a sequence $\left(g_{n}\right)_{n \in \mathbb{N}} \in \mathrm{S}_{C(K)}$ with $\left\|f \cdot g_{n}\right\|-$ $\left|f(x) g_{n}(x)\right| \rightarrow 0$ as $n \rightarrow \infty$. We will distinguish two cases:

1. $f(x)=0$. In this case, $f$ is not invertible and we find a sequence $\left(g_{n}\right)_{n \in \mathbb{N}} \subseteq \mathrm{S}_{C(K)}$ such that $\left\|f \cdot g_{n}\right\| \rightarrow 0$ as $n \rightarrow \infty$, which implies that $\left(\left\|f \cdot g_{n}\right\|-\left|f(x) g_{n}(x)\right|\right)_{n \in \mathbb{N}}$ also converges to 0 . 
2. $f(x) \neq 0$. If $\|f\|_{\infty}=|f(x)|$, then we simply take $g_{n}=1$ for all $n \in \mathbb{N}$. Suppose now that $|f(x)|<\|f\|_{\infty}$. For $n \in \mathbb{N}$ sufficiently large we define the continuous function

$$
\begin{aligned}
h_{n}: F_{n} & \rightarrow \mathbb{K} \\
y & \mapsto h_{n}(y):= \begin{cases}0 & y \in|f|^{-1}\left(\left[|f(x)|+\frac{1}{n},\|f\|_{\infty}\right]\right) \\
1 & y \in|f|^{-1}([0,|f(x)|])\end{cases}
\end{aligned}
$$

where $F_{n}:=|f|^{-1}\left(\left[|f(x)|+\frac{1}{n},\|f\|_{\infty}\right]\right) \cup f^{-1}([0,|f(x)|])$ is a union of nonempty disjoint closed subsets of $K$. Let $g_{n}$ be a continuous extension of $h_{n}$ in such a way that $\left\|g_{n}\right\|=\left\|h_{n}\right\|=1$. Since

$$
\left|f(x) g_{n}(x)\right| \leq\left\|f \cdot g_{n}\right\|_{\infty} \leq|f(x)|+\frac{1}{n}=\left|f(x) g_{n}(x)\right|+\frac{1}{n},
$$

it is easy to see that $\left(\left\|f \cdot g_{n}\right\|-\left|f(x) g_{n}(x)\right|\right)_{n \in \mathbb{N}}$ converges to 0 .

Finally, we study the set of topological divisors of zero of (completely) uniform algebras:

Theorem 4 Let $A$ be a uniform algebra. Then:

$$
t \ell d(0) \subseteq\left\{a \in A:\|a\|_{M_{A} \min }=0\right\} .
$$

In addition, if $A$ is a completely uniform algebra, then:

$$
t \ell d(0)=\left\{a \in A:\|a\|_{M_{A} \min }=0\right\} .
$$

Proof Let $a \in t \ell d(0)$. There exists a sequence $\left(b_{n}\right)_{n \in \mathbb{N}} \subset \mathrm{S}_{A}$ such that $\left\|a b_{n}\right\| \rightarrow 0$ as $n \rightarrow \infty$. Since $\left\|b_{n}\right\|_{M_{A}}=\left\|b_{n}\right\|=1$ for every $n \in \mathbb{N}$, we can choose $\phi_{n} \in M_{A}$ in such a way that $\left|\phi_{n}\left(b_{n}\right)\right| \rightarrow 1$ as $n \rightarrow \infty$. Observe that $\left|\phi_{n}\left(a b_{n}\right)\right| \leq\left\|a b_{n}\right\|$ for every $n \in \mathbb{N}$, thus $\left|\phi_{n}\left(a b_{n}\right)\right| \rightarrow 0$ as $n \rightarrow \infty$. Now $\left|\phi_{n}\left(a b_{n}\right)\right|=\left|\phi_{n}(a)\right|\left|\phi_{n}\left(b_{n}\right)\right|$ for every $n \in \mathbb{N}$, which neccesarily implies that $\left|\phi_{n}(a)\right| \rightarrow 0$ as $n \rightarrow \infty$. As a consequence, $\|a\|_{M_{A} \min }=0$. Conversely, assume that $A$ is completely uniform. Take $a \in A$ such that $\|a\|_{M_{A} \text { min }}=0$. There exists $\left(\phi_{n}\right)_{n \in \mathbb{N}} \subset M_{A}$ such that $\phi_{n}(a) \rightarrow 0$ when $n \rightarrow \infty$. By hypothesis, for every $n \in \mathbb{N}$ we can find $b_{n} \in \mathrm{S}_{A}$ such that $\left\|a b_{n}\right\|-\left|\phi_{n}\left(a b_{n}\right)\right|<\frac{1}{n}$. Since $\left|\phi_{n}\left(b_{n}\right)\right| \leq 1$ for every $n \in \mathbb{N}$, we conclude that $\left\|a b_{n}\right\| \rightarrow 0$ as $n \rightarrow \infty$.

\subsection{Spectra of an algebra}

Our purpose in this subsection is to extend the notions of the point, residual and continuous spectra to the scope of algebras and prove that the spectral decomposition theorem also holds for them.

The spectrum of an element $a$ of an algebra $A$ is defined as $\sigma(a):=\{\lambda \in \mathbb{C}: a-\lambda 1 \notin$ $\mathcal{U}(A)\}$. It is well known that $\sigma(a)=\left\{\phi(a): \phi \in M_{A}\right\}$.

For a linear operator $T: X \rightarrow X$ on a normed space $X, \sigma(T)$ is a non-void compact subset of $\mathbb{C}$ which can be decomposed into the point spectrum, the continuous spectrum and the residual spectrum as $\sigma(T)=\sigma_{p}(T) \cup \sigma_{c}(T) \cup \sigma_{r}(T)$.

According to Theorem 1, the point spectrum of a linear operator $T: X \rightarrow X$ on a normed space $X$ satisfies that $\sigma_{p}(T):=\{\lambda \in \mathbb{C}: \operatorname{ker}(T-\lambda I) \neq\{0\}\}=\{\lambda \in \mathbb{C}: T-\lambda I \in \ell d(0)\}$. This motivates the following definition:

Definition 2 Given an element $a$ of an algebra $A$, the point spectrum of $a$ is defined as:

$$
\sigma_{p}(a):=\{\lambda \in \mathbb{C}: a-\lambda 1 \in \ell d(0)\} .
$$


Since $U(A) \cap \ell d(0)=\varnothing$, we conclude that $\sigma_{p}(a) \subseteq \sigma(a)$. It is also clear that $\ell d(0)=$ $\left\{a \in A: 0 \in \sigma_{p}(a)\right\}$.

According to Theorem 1, Remark 1 and the Open Mapping Theorem, the continuous spectrum of a linear operator $T: X \rightarrow X$ on a Banach space $X$ satisfies that

$$
\begin{aligned}
\sigma_{c}(T) & :=\{\lambda \in \mathbb{C}: \operatorname{ker}(T-\lambda I)=\{0\} \text { and }(T-\lambda I)(X) \subsetneq \overline{(T-\lambda I)(X)}=X\} \\
& =\left\{\lambda \in \mathbb{C}: \operatorname{ker}(T-\lambda I)=\{0\}, T-\lambda I \notin \mathcal{U}(\mathcal{B}(X)) \& \operatorname{ker}\left((T-\lambda I)^{*}\right)=\{0\}\right\} \\
& =\left\{\lambda \in \mathbb{C}: T-\lambda I \notin \ell d(0) \cup \mathcal{U}(\mathcal{B}(X)) \text { and }(T-\lambda I)^{*} \notin \ell d(0)\right\} .
\end{aligned}
$$

Accordingly, we define the continuous spectrum as follows:

Definition 3 Given an element $a$ of a $*$-algebra $A$, the continuous spectrum of $a$ is defined as:

$$
\sigma_{c}(a):=\left\{\lambda \in \mathbb{C}: a-\lambda 1 \notin \ell d(0) \cup \mathcal{U}(A) \text { and }(a-\lambda 1)^{*} \notin \ell d(0)\right\} .
$$

Recall that in a $*$-ring $R$, an element $r \in R$ satisfies that $r^{*} \in \ell d(0)$ if and only if $r \in r d(0)$. This fact implies that:

$$
\sigma_{c}(a)=\{\lambda \in \mathbb{C}: a-\lambda 1 \notin \ell d(0) \cup \mathcal{U}(A) \cup \operatorname{rd}(0)\} .
$$

The last equality allows to extend the continuous spectrum to algebras not necessarily endowed with an involution. Observe that by definition, $\sigma_{c}(a) \subseteq \sigma(a)$.

According to Theorem 1, Remark 1 and the Open Mapping Theorem, the residual spectrum of a continuous linear operator $T: X \rightarrow X$ on a Banach space $X$ satisfies that:

$$
\begin{aligned}
\sigma_{r}(T) & =\left\{\lambda \in \mathbb{C}: \operatorname{ker}(T-\lambda I)=\{0\} \text { and } \operatorname{ker}\left((T-\lambda I)^{*}\right) \neq\{0\}\right\} \\
& =\left\{\lambda \in \mathbb{C}: T-\lambda I \notin \ell d(0) \text { and }(T-\lambda I)^{*} \in \ell d(0)\right\} .
\end{aligned}
$$

This fact motivates the following definition:

Definition 4 Given an element $a$ of a $*$-algebra $A$, the residual spectrum of $a$ is defined as

$$
\sigma_{r}(a):=\left\{\lambda \in \mathbb{C}: a-\lambda 1 \notin \ell d(0) \text { and }(a-\lambda 1)^{*} \in \ell d(0)\right\} .
$$

By using again that in a $*$-ring $R$, an element $r \in R$ satisfies that $r^{*} \in \ell d(0)$ if and only if $r \in r d(0)$, we conclude that

$$
\sigma_{r}(a)=\{\lambda \in \mathbb{C}: a-\lambda 1 \in \operatorname{rd}(0) \backslash \ell d(0)\} .
$$

The last equality allows us to extend the residual spectrum to algebras not necessarily endowed with an involution. Since $\mathcal{U}(A) \cap r d(0)=\varnothing$, we have that $\sigma_{r}(a) \subseteq \sigma(a)$.

We now show that the spectrum of an element in an algebra can be decomposed as the disjoint union of the point spectrum, the continuous spectrum and the residual spectrum.

Theorem 5 Let $A$ be an algebra. Then $\left\{\sigma_{p}(a), \sigma_{c}(a), \sigma_{r}(a)\right\}$ is a partition of $\sigma(a)$.

Proof We already know that $\sigma(a) \supseteq \sigma_{p}(a) \cup \sigma_{c}(a) \cup \sigma_{r}(a)$. Let $\lambda \in \mathbb{C}$. If $a-\lambda 1 \in \ell d(0)$, then $\lambda \in \sigma_{p}(a)$. Otherwise, we have two possibilities: if $a-\lambda 1 \in r d(0)$, then $\lambda \in \sigma_{r}(a)$, and if $a-\lambda 1 \notin r d(0)$, then $\lambda \in \sigma_{c}(a)$. Finally it is clear that $\sigma_{p}(a), \sigma_{c}(a)$ and $\sigma_{r}(a)$ are pairwise disjoint.

Remark 2 We point out that the notion of left point spectrum $\pi_{l}(a)$ introduced in [8, Def. Chapter I] corresponds to the definition of $\sigma_{p}(a)$ we have generalized from the Banach algebra of operators $\mathcal{B}(X)$ to the scope of algebras. 
In order to illustrate our results we provide an example of the spectral decomposition in other algebras different from the operator algebras and we show such a decomposition for $C(K)$. Recall that the algebraic support of a real or complex valued function $f \in C(K)$ is given by $\underline{\operatorname{supp}(f)}:=\{x \in K: f(x) \neq 0\}$ and the topological support is given by $\overline{\operatorname{supp}}(f):=\overline{\operatorname{supp}(f)}$.

Theorem 6 Let $K$ is a Hausdorff compact space. If $f \in C(K)$ then $\sigma_{r}(f)=\varnothing$,

$$
\begin{aligned}
& \sigma_{p}(f)=\left\{f(x): x \in K \text { and int }\left(f^{-1}(\{f(x)\})\right) \neq \varnothing\right\} \text { and } \\
& \sigma_{c}(f)=\left\{f(x): x \in K \text { and } \operatorname{int}\left(f^{-1}(\{f(x)\})\right)=\varnothing\right\} .
\end{aligned}
$$

Proof It is well-known that $\mathcal{U}(C(K))=\{f \in C(K): \operatorname{supp}(f)=K\}$, therefore $\sigma(f)=$ $\{f(x): x \in K\}$ for every $f \in C(K)$. Now, let $x \in K$ with $f(x) \in \sigma_{p}(f)$. Then $f-f(x) 1 \in$ $\ell d(0)$. We point out that $\ell d(0)=r d(0)=\{f \in C(K): \overline{\operatorname{supp}}(f) \subsetneq K\}$. Indeed, given $f \in C(K)$ such that $\operatorname{supp}(f)$ is not dense in $K$, by the Uryshon's Lemma we can find $g \in C(K) \backslash\{0\}$ such that $g(\overline{\operatorname{supp}}(f))=\{0\}$. Conversely, if $f \in \ell d(0)$ and $\overline{\operatorname{supp}}(f)=K$, then there exists $g \in C(K) \backslash\{0\}$ such that $f \cdot g=0$, but then $K=\overline{\operatorname{supp}}(f) \subseteq \overline{g^{-1}(\{0\})}=$ $g^{-1}(\{0\})$, which is a contradiction. Consequently, we have that $\left.\overline{\operatorname{supp}}(f-f(x) 1)\right) \subsetneq K$. Note that $\operatorname{supp}(f-f(x) 1))=K \backslash f^{-1}(\{f(x)\})$, and then $K \backslash f^{-1}(\{f(x)\})$ is not dense in $K$ which is equivalent to the fact that int $\left(f^{-1}(\{f(x)\})\right) \neq \varnothing$. We obtain

$$
\begin{aligned}
\sigma_{p}(f) & :=\{f(x): x \in K \text { and } f-f(x) 1 \in \ell d(0)\} \\
& =\{f(x): x \in K \text { and } \operatorname{supp}(f-f(x) 1)) \subsetneq K\} \\
& =\left\{f(x): x \in K \text { and } K \backslash f^{-1}(\{f(x)\}) \text { is not dense in } K\right\} \\
& =\left\{f(x): x \in K \text { and int }\left(f^{-1}(\{f(x)\})\right) \neq \varnothing\right\} .
\end{aligned}
$$

Note that if $K$ is a Hausdorff compact space, then $C(K)$ is a $*$-algebra with the involution given by the complex conjugation. It is also obvious that $f \in C(K)$ is a divisor of 0 if and only if so is $\bar{f}$. Therefore,

$$
\begin{aligned}
\sigma_{c}(f) & :=\{f(x): x \in K \text { and } f-f(x) 1 \notin \ell d(0) \text { and } \overline{f-f(x) 1} \notin \ell d(0)\} \\
& =\{f(x): x \in K \text { and } f-f(x) 1 \notin \ell d(0)\} \\
& =\left\{f(x): x \in K \text { and int }\left(f^{-1}(\{f(x)\})\right)=\varnothing\right\} .
\end{aligned}
$$

Finally observe that the spectral decomposition theorem and the fact that $\sigma_{p}(f) \cup \sigma_{c}(f)=$ $\sigma(f)$ imply that $\sigma_{r}(f)=\varnothing$.

Notice that if $K$ is finite, $\sigma_{c}(f)=\varnothing$ and $\sigma(f)=\sigma_{p}(f)$ for any $f \in C(K)$.

We finally study the approximated spectrum of elements in an algebra. The approximated spectrum of a continuous linear operator $T: X \rightarrow X$ on a normed space $X$ is defined as

$$
\sigma_{a}(T):=\left\{\lambda \in \mathbb{C}: \exists\left(x_{n}\right)_{n \in \mathbb{N}} \subseteq \mathrm{S}_{X} \quad(T-\lambda I)\left(x_{n}\right) \rightarrow 0\right\} .
$$

By taking into consideration Theorem 2, we have that

$$
\begin{aligned}
\sigma_{a}(T) & :=\left\{\lambda \in \mathbb{C}: \exists\left(x_{n}\right)_{n \in \mathbb{N}} \subseteq \mathrm{S}_{X}(T-\lambda I)\left(x_{n}\right) \rightarrow 0\right\} \\
& =\left\{\lambda \in \mathbb{C}:\|T-\lambda I\|_{\min }=0\right\} \\
& =\{\lambda \in \mathbb{C}: T-\lambda I \in \operatorname{t\ell d}(0)\} .
\end{aligned}
$$

This notion is extended to normed algebras in [8]. 
Definition 5 Given an element $a$ of a normed algebra $A$, the approximated spectrum of $a$ is defined as

$$
\sigma_{a}(a):=\{\lambda \in \mathbb{C}: a-\lambda 1 \in t \ell d(0)\}=\left\{\lambda \in \mathbb{C}:\|a-\lambda 1\|_{\ell 1}=0\right\} .
$$

It is clear that $\sigma_{p}(a) \subseteq \sigma_{a}(a)$. Since $\mathcal{U}(A) \cap t \ell d(0)=\varnothing$, we conclude that $\sigma_{a}(a) \subseteq \sigma(a)$.

As a direct consequence of Theorem 4 we obtain the following reformulation of the approximated spectrum in uniform algebras:

Theorem 7 If $A$ is a uniform algebra, then $\sigma_{a}(x) \subseteq\left\{\lambda \in \mathbb{C}:\|x-\lambda 1\|_{M_{A} \min }=0\right\}$. If, in addition, $A$ is completely uniform, then $\sigma_{a}(x)=\left\{\lambda \in \mathbb{C}:\|x-\lambda 1\|_{M_{A} \min }=0\right\}$.

In the case of $C(K)$ the approximated spectrum states as follows:

Proposition 4 Let $K$ be a Hausdorff compact space. If $f \in C(K)$, then $\sigma_{a}(f)=\sigma(f)$.

Proof We first point out that $t \ell d(0)=C(K) \backslash \mathcal{U}(C(K))$. Indeed, let $f \in C(K) \backslash \mathcal{U}(C(K))$. Since 0 is clearly a topological divisor of 0 , we may assume that $f \neq 0$. For $n \in \mathbb{N}$ sufficiently large we can consider the continuous function

$$
\begin{aligned}
h_{n}: F_{n} & \rightarrow \mathbb{K} \\
x & \mapsto h_{n}(x):= \begin{cases}0 & x \in|f|^{-1}\left(\left[\frac{1}{n},\|f\|_{\infty}\right]\right) \\
1 & x \in f^{-1}(\{0\})\end{cases}
\end{aligned}
$$

where $F_{n}:=|f|^{-1}\left(\left[\frac{1}{n},\|f\|_{\infty}\right]\right) \cup f^{-1}(\{0\})$ is a union of nonempty disjoint closed subsets of $K$. Since $K$ is a normal topological space, Tietze's Extension Theorem assures the existence of $g_{n} \in C(K)$ such that $\left\|g_{n}\right\|_{\infty}=\left\|h_{n}\right\|_{\infty}=1$ and $\left.g_{n}\right|_{F}=h_{n}$. Finally, if $x \in|f|^{-1}\left(\left[\frac{1}{n},\|f\|_{\infty}\right]\right)$, then $\left|\left(f \cdot g_{n}\right)(x)\right|=0$ and if $x \in K \backslash|f|^{-1}\left(\left[\frac{1}{n},\|f\|_{\infty}\right]\right)=$ $|f|^{-1}\left(\left[0, \frac{1}{n}\right)\right)$, then $\left|\left(f \cdot g_{n}\right)(x)\right|<\frac{1}{n}$. As a consequence, $\left\|f \cdot g_{n}\right\| \leq \frac{1}{n}$. Consequently, we obtain that

$$
\begin{aligned}
\sigma_{a}(f) & =\{f(x): x \in K \text { and } f-f(x) 1 \in t \ell d(0)\} \\
& =\{f(x): x \in K \text { and } f-f(x) 1 \in C(K) \backslash \mathcal{U}(C(K))\} \\
& =\{f(x): x \in K\}=\sigma(f) .
\end{aligned}
$$

Notice that if $K$ is finite, $\sigma_{c}(f)=\varnothing$ and $\sigma(f)=\sigma_{p}(f)=\sigma_{a}(f)$ for any $f \in C(K)$.

\subsection{Invertibility in topological rings}

In a Hausdorff topological ring $R$ if an element $r \in R$ satisfies that $\sum_{n=0}^{\infty} r^{n}$ is convergent, then $1-r \in \mathcal{U}(R)$ and $(1-r)^{-1}=\sum_{n=0}^{\infty} r^{n}$. Indeed, this can be seen by taking into consideration that

$$
(1-r) \sum_{n=0}^{k} r^{n}=\left(\sum_{n=0}^{k} r^{n}\right)(1-r)=1-r^{k+1}
$$

for all $k \in \mathbb{N}$. A topological ring $R$ is called practical provided that $0 \in \operatorname{cl}(\mathcal{U}(R))$. We refer the reader to $[19,22-25]$ for a wider perspective on practical rings. Every normed algebra 
is trivially a practical ring. Practical rings are crucial at this point because they allow us to extend the concept of derivative from real or complex topological vector spaces to topological modules over practical rings as follows:

Definition 6 A function $f: M \rightarrow N$ between topological (left) modules $M$ and $N$ over a practical ring $R$ is differentiable at $m \in M$ in the direction of $l \in M$ provided that the function

$$
\begin{aligned}
\mathcal{U}(R) & \rightarrow N \\
r & \mapsto r^{-1}(f(m+r l)-f(m))
\end{aligned}
$$

has a unique limit at 0 . The limit is usually denoted by $\mathrm{D}_{l} f(m)$ or $\frac{\partial f}{\partial l}(m)$.

In [7, Theorem 1.1.23], it is shown that the inversion map on the group of invertibles of a Banach algebra is $\mathbb{C}$-differentiable. In the next theorem we generalize this result to complete normed rings using a stronger derivative than the real or complex one. It is worth mentioning that our proof uses a different technique from the one employed in [7, Theorem 1.1.23].

Theorem 8 Let $R$ be a complete normed ring. Let $r \in \mathcal{U}(R)$. Every $s \in U_{R}\left(r,\left\|r^{-1}\right\|^{-1}\right)$ satisfies that $s \in \mathcal{U}(R)$ and

$$
s^{-1}=r^{-1} \sum_{n=0}^{\infty}\left(1-s r^{-1}\right)^{n} \text { and }\left\|s^{-1}\right\| \leq \frac{\left\|r^{-1}\right\|}{1-\|r-s\|\left\|r^{-1}\right\|} .
$$

In addition,

$$
\left\|r^{-1}-s^{-1}\right\| \leq \frac{\left\|r^{-1}\right\|^{2}}{1-\left\|r^{-1}\right\|\|s-r\|}\|s-r\|
$$

so $U(R)$ is open and the inversion map on $U(R)$ given by $u \mapsto u^{-1}$ is continuous. Moreover, if $0 \in \mathrm{cl}(\mathcal{U}(R))$, then the inversion map is differentiable at every $u \in Z(U(R))$ in the direction of any $v \in R$ being its derivative equal to $-u^{-1} v u^{-1}$.

Proof First of all, since $\|r-s\|<\left\|r^{-1}\right\|^{-1}$, then $\left\|1-s r^{-1}\right\| \leq\|r-s\|\left\|r^{-1}\right\|<1$ and hence $\sum_{n=0}^{\infty}\left(1-s r^{-1}\right)^{n}$ is absolutely convergent, so it is convergent. As a consequence, $s r^{-1} \in \mathcal{U}(R)$ and so does $s$. It follows that $\left(s r^{-1}\right)^{-1}=\sum_{n=0}^{\infty}\left(1-s r^{-1}\right)^{n}$ and thus $s^{-1}=$ $r^{-1} \sum_{n=0}^{\infty}\left(1-s r^{-1}\right)^{n}$. Therefore

$$
\begin{aligned}
\left\|s^{-1}\right\| & \leq\left\|r^{-1}\right\| \sum_{n=0}^{\infty}\left\|1-s r^{-1}\right\|^{n} \\
& \leq\left\|r^{-1}\right\| \sum_{n=0}^{\infty}\left(\|r-s\|\left\|r^{-1}\right\|\right)^{n}=\frac{\left\|r^{-1}\right\|}{1-\|r-s\|\left\|r^{-1}\right\|} .
\end{aligned}
$$

Now

$$
\begin{aligned}
\left\|r^{-1}-s^{-1}\right\| & =\left\|r^{-1}(s-r) s^{-1}\right\| \leq\left\|r^{-1}\right\|\|s-r\|\left\|s^{-1}\right\| \\
& \leq\left\|r^{-1}\right\|\|s-r\| \frac{\left\|r^{-1}\right\|}{1-\|r-s\|\left\|r^{-1}\right\|}=\frac{\left\|r^{-1}\right\|^{2}}{1-\left\|r^{-1}\right\|\|s-r\|}\|s-r\| .
\end{aligned}
$$

This proves the continuity of the inversion map at $r$. Finally, let us show the differentiablity of the inversion map. Let $u, w \in \mathcal{U}(R)$ and $v \in R$. If $\|w\|<\|v\|^{-1}\left\|u^{-1}\right\|^{-1}$, then $\left\|-w v u^{-1}\right\|=$ 
$\left\|w v u^{-1}\right\| \leq\|w\|\|v\|\left\|u^{-1}\right\|<1$, therefore $1+w v u^{-1} \in \mathcal{U}(R)$ and $\left(1+w v u^{-1}\right)^{-1}=$ $\sum_{n=0}^{\infty}\left(-w v u^{-1}\right)^{n}$. Hence

$$
\begin{aligned}
& w^{-1}\left((u+w v)^{-1}-u^{-1}\right)=w^{-1}\left(u^{-1}\left(1+w v u^{-1}\right)^{-1}-u^{-1}\right) \\
& =w^{-1} u^{-1}\left(\left(1+w v u^{-1}\right)^{-1}-1\right)=w^{-1} u^{-1}\left(\sum_{n=0}^{\infty}\left(-w v u^{-1}\right)^{n}-1\right) \\
& =w^{-1} u^{-1} \sum_{n=1}^{\infty}\left(-w v u^{-1}\right)^{n}=w^{-1} u^{-1}\left(-w v u^{-1}\right) \sum_{n=0}^{\infty}\left(-w v u^{-1}\right)^{n} \\
& =u^{-1} w^{-1}\left(-w v u^{-1}\right) \sum_{n=0}^{\infty}\left(-w v u^{-1}\right)^{n}=-u^{-1} v u^{-1} \sum_{n=0}^{\infty}\left(-w v u^{-1}\right)^{n} \\
& =-u^{-1} v u^{-1}-u^{-1} v u^{-1} \sum_{n=1}^{\infty}\left(-w v u^{-1}\right)^{n} .
\end{aligned}
$$

Finally,

$$
\begin{aligned}
\left\|-u^{-1} v u^{-1} \sum_{n=1}^{\infty}\left(-w v u^{-1}\right)^{n}\right\| & \leq\|v\|\left\|u^{-1}\right\|^{2} \sum_{n=1}^{\infty}\left(\|w\|\|v\|\left\|u^{-1}\right\|\right)^{n} \\
& =\|v\|\left\|u^{-1}\right\|^{2} \frac{\|w\|\|v\|\left\|u^{-1}\right\|}{1-\|w\|\|v\|\left\|u^{-1}\right\|} \rightarrow 0
\end{aligned}
$$

as $w \rightarrow 0$, which implies that $\lim _{w \rightarrow 0} w^{-1}\left((u+w v)^{-1}-u^{-1}\right)=-u^{-1} v u^{-1}$.

A simple version of Theorem 8 for topological rings follows.

Theorem 9 Let $R$ be a Hausdorff topological ring. Let $S$ be a subring of $R$ such that $U(S) \subseteq$ $Z(\mathcal{U}(R))$. If $0 \in \operatorname{cl}(\mathcal{U}(S)), \mathcal{U}(R)$ is open and the inversion map on $R$ is continuous, then the inversion map on $R$ is $S$-differentiable and its derivative at $u \in \mathcal{U}(R)$ in the direction of $v \in R$ is given by $-u^{-1} v u^{-1}$.

Proof Fix arbitrary elements $u \in \mathcal{U}(R)$ and $v \in R$. For every $s \in \mathcal{U}(S)$ sufficiently close to 0 we have that $u+s v \in \mathcal{U}(R)$ and

$$
\begin{aligned}
s^{-1}\left((u+s v)^{-1}-u^{-1}\right) & =s^{-1}(u+s v)^{-1}(-s v) u^{-1} \\
& =-(u+s v)^{-1} v u^{-1} \rightarrow-u^{-1} v u^{-1}
\end{aligned}
$$

as $s \rightarrow 0$.

In [9, Proposition 1.2.28] it was shown that every element in the boundary of the invertibles in a Banach algebra is a topological zero divisor. As far as we know, Banach algebras are the only topological rings known to satisfy such property. In this subsection, we will extend this result to a new class of normed rings: complete almost absolutely normed rings. This is a new concept in Ring Theory. However, in order to motivate this new notion, we need to introduce another new concept: absolutely invertibles.

Definition 7 (Absolutely invertible) Let $R$ be a semi-normed ring. We say that $u \in \mathcal{U}(R)$ is absolutely invertible provided that $\left\|u^{-1}\right\|=\|u\|^{-1}$. The subset of absolutely invertibles of $R$ is denoted by $\mathcal{U}_{1}(R)$. 
Observe that every absolutely semivalued ring $R$ satisfies that $\mathcal{U}_{1}(R)=\mathcal{U}(R)$. The following technical lemma relies in the inequality $\|a\|\left\|b^{-1}\right\|^{-1} \leq\|a b\| \leq\|a\|\|b\|$, that holds for all $a \in R$ and $b \in \mathcal{U}(R)$ in every seminormed ring $R$.

Lemma 1 Let $R$ be a semi-normed ring. Let $u, v \in R$. Then:

1. If $u \in \mathcal{U}_{1}(R)$, then $-u, u^{-1} \in \mathcal{U}_{1}(R)$.

2. If $v \in \mathcal{U}_{1}(R)$, then $\|u v\|=\|v u\|=\|u\|\|v\|$.

3. If $u, v \in \mathcal{U}_{1}(R)$, then $u v \in \mathcal{U}_{1}(R)$.

4. $\|1\|=1$ and only if $1 \in \mathcal{U}_{1}(R)$.

5. If $\mathcal{U}_{1}(R) \neq \varnothing$, then $\|1\|=1$.

6. If $v \in \mathcal{U}(R),\|v\| \leq\|u\|$ and $\left\|v^{-1}\right\| \leq\|u\|^{-1}$, then $v \in \mathcal{U}_{1}(R),\|u\|=\|v\|$ and $\left\|v^{-1}\right\|=\|u\|^{-1}$.

7. If $R$ is $a *$-ring and the involution is an isometry, then $\mathcal{U}_{1}(R)^{*}=\mathcal{U}_{1}(R)$.

In particular, $\mathfrak{U}_{1}(R)$ is a subgroup of $\mathcal{U}(R)$ if $R$ is unital.

Proof 1. Trivial.

2. Notice that $\|u\|\|v\|=\|u\|\left\|v^{-1}\right\|^{-1} \leq\|u v\| \leq\|u\|\|v\|$. Similarly, $\|v u\|=\|u\|\|v\|$.

3. $\left\|(u v)^{-1}\right\|=\left\|v^{-1} u^{-1}\right\|=\left\|v^{-1}\right\|\left\|u^{-1}\right\|=\|v\|^{-1}\|u\|^{-1}=(\|v\|\|u\|)^{-1}=\|u v\|^{-1}$.

4. If $\|1\|=1$, then it is trivial that $1 \in \mathcal{U}_{1}(R)$. Conversely, if $1 \in \mathcal{U}_{1}(R)$, then $\|1\|=\|1\|^{-1}$ so $\|1\|=1$.

5. Fix an arbitrary $v \in \mathcal{U}_{1}(R)$, so $\|1\|=\left\|v v^{-1}\right\|=\|v\|\left\|v^{-1}\right\|=\|v\|\|v\|^{-1}=1$.

6. On the one hand, $\|u\|^{-1} \leq\|v\|^{-1} \leq\left\|v^{-1}\right\| \leq\|u\|^{-1}$. Thus, $\|v\|^{-1}=\left\|v^{-1}\right\|$, so $v \in \mathcal{U}_{1}(R)$. Then $\|v\|^{-1}=\left\|v^{-1}\right\| \leq\|u\|^{-1}$, meaning that $\|u\| \leq\|v\|$. Since $\|v\| \leq\|u\|$ by hypothesis, we conclude that $\|v\|=\|u\|$. Finally, $\left\|v^{-1}\right\|=\|v\|^{-1}=\|u\|^{-1}$.

7. Fix any arbitrary $r \in \mathcal{U}_{1}(R)$. Observe that $\left(r^{*}\right)^{-1}=\left(r^{-1}\right)^{*}$, so

$$
\left\|\left(r^{*}\right)^{-1}\right\|=\left\|\left(r^{-1}\right)^{*}\right\|=\left\|r^{-1}\right\|=\|r\|^{-1}=\left\|r^{*}\right\|^{-1},
$$

hence $r^{*} \in \mathcal{U}_{1}(R)$.

In the next example we characterize the set of absolutely invertibles of the algebra of bounded operators on a normed space, which corresponds to the surjective linear isometries. We include its proof for the sake of completeness.

Example 2 Given a normed space $X$, the set of of absolutely invertibles in $\mathcal{B}(X)$ is given by:

$$
\mathcal{U}_{1}(\mathcal{B}(X))=\{\lambda T: \lambda>0, T: X \rightarrow X \text { is a surjective linear isometry }\} .
$$

Proof Indeed, if $T: X \rightarrow X$ is a surjective linear isometry and $\lambda>0$, then $\|\lambda T\|=\lambda$ and $\left\|(\lambda T)^{-1}\right\|=\left\|\lambda^{-1} T^{-1}\right\|=\lambda^{-1}\left\|T^{-1}\right\|=\lambda^{-1}=\|\lambda T\|^{-1}$. As a consequence, $\lambda T \in$ $\mathcal{U}_{1}(\mathcal{B}(X))$. Conversely, let $S \in \mathcal{U}_{1}(\mathcal{B}(X))$. We will prove that $T:=\frac{S}{\|S\|}$ is a surjective linear isometry. Observe that $T \in \mathcal{U}_{1}(\mathcal{B}(X))$. Indeed,

$$
\left\|T^{-1}\right\|=\frac{\left\|S^{-1}\right\|}{\|S\|^{-1}}=1=\|T\|^{-1} .
$$

We point out that $T\left(\mathrm{~B}_{X}\right) \subseteq \mathrm{B}_{X}$ and $T^{-1}\left(\mathrm{~B}_{X}\right) \subseteq \mathrm{B}_{X}$ because $\|T\|=1=\left\|T^{-1}\right\|$. This shows that $T\left(\mathrm{~B}_{X}\right)=\mathrm{B}_{X}$. Since $T$ is an isomorphism, it must occur that $T\left(\mathrm{U}_{X}\right)=\mathrm{U}_{X}$ and $T\left(\mathrm{~S}_{X}\right)=\mathrm{S}_{X}$. As a consequence, $T$ is an isometry. 
Theorem 10 Let $H$ be a Hilbert space with $\operatorname{dim}(H) \geq 2$. Then $\mathcal{U}_{1}(\mathcal{B}(H))$ is not a normal subgroup of $U(\mathcal{B}(H))$.

Proof Since $\operatorname{dim}(H) \geq 2$, we can find $x, y \in \mathrm{S}_{H}$ orthogonal. Denote $K:=\operatorname{span}\{x, y\}$. Note that $H=K \oplus_{2} K^{\perp}$ so every element of $H$ is uniquely of the form $\alpha x+\beta y+k^{\perp}$ for some $\alpha, \beta \in \mathbb{K}$ and $k^{\perp} \in K^{\perp}$. Consider the following surjective linear isometry

$$
\begin{aligned}
H: & \rightarrow H \\
\alpha x+\beta y+k^{\perp} & \mapsto S\left(\alpha x+\beta y+k^{\perp}\right):=\beta x+\alpha y+k^{\perp} .
\end{aligned}
$$

Next, take the following isomorphism

$$
\begin{aligned}
T: H & \rightarrow H \\
\alpha x+\beta y+k^{\perp} & \mapsto T\left(\alpha x+\beta y+k^{\perp}\right):=2 \alpha x+3 \beta y+k^{\perp} .
\end{aligned}
$$

Observe that the inverse of $T$ is given by

$$
\begin{aligned}
T^{-1}: & \rightarrow H \\
\alpha x+\beta y+k^{\perp} & \mapsto T^{-1}\left(\alpha x+\beta y+k^{\perp}\right):=\frac{\alpha}{2} x+\frac{\beta}{3} y+k^{\perp} .
\end{aligned}
$$

Suppose to the contrary that $\mathcal{U}_{1}(\mathcal{B}(H))$ is a normal subgroup of $\mathcal{U}(\mathcal{B}(H))$. Then $T^{-1} \circ S \circ T$ is a positive multiple of a surjective linear isometry in view of Example 2. So, there exists a surjective linear isometry $R: H \rightarrow H$ and $\lambda>0$ such that $T^{-1} \circ S \circ T=\lambda R$. This means that

$$
\left\|\left(T^{-1} \circ S \circ T\right)(z)\right\|=\lambda\|R(z)\|=\lambda\|z\|
$$

for all $z \in H$. In particular, the quotient

$$
\frac{\left\|\left(T^{-1} \circ S \circ T\right)(z)\right\|^{2}}{\|z\|^{2}}
$$

is constant $\left(\lambda^{2}\right)$ for all $z \in H \backslash\{0\}$. Next, for every $\alpha, \beta \in \mathbb{K}$ not both zero,

$$
\frac{\left\|\left(T^{-1} \circ S \circ T\right)(\alpha x+\beta y)\right\|^{2}}{\|\alpha x+\beta y\|^{2}}=\frac{\frac{9|\beta|^{2}}{4}+\frac{4|\alpha|^{2}}{9}}{\alpha^{2}+\beta^{2}}
$$

and

$$
\frac{\left\|\left(T^{-1} \circ S \circ T\right)(\beta x+\alpha y)\right\|^{2}}{\|\beta x+\alpha y\|^{2}}=\frac{\frac{9|\alpha|^{2}}{4}+\frac{4|\beta|^{2}}{9}}{\beta^{2}+\alpha^{2}} .
$$

Since (4) and (5) must be equal, we obtain that

$$
\frac{9|\beta|^{2}}{4}+\frac{4|\alpha|^{2}}{9}=\frac{9|\alpha|^{2}}{4}+\frac{4|\beta|^{2}}{9},
$$

in other words,

$$
\left(\frac{9}{4}-\frac{4}{9}\right)|\beta|^{2}=\left(\frac{9}{4}-\frac{4}{9}\right)|\alpha|^{2},
$$

meaning that $|\beta|=|\alpha|$, which does not necessarily have to occur. Thus, we have reached a contradiction and $T^{-1} \circ S \circ T$ is not a multiple of any surjective linear isometry on $H$. This implies the result. 
It is not difficult to see that the absolutely invertibles in the algebra of continuous functions $C(K)$ are exactly the invertibles.

Example 3 In every Hausdorff compact topological space $X, \mathcal{U}_{1}(C(K))=\mathcal{U}(C(K))$.

Definition 8 (Absolutely semi-normed ring) A semi-normed (normed) ring $R$ is called:

- Absolutely semi-normed (normed) if $\mathfrak{U}_{1}(R)=\mathcal{U}(R)$.

- Almost absolutely semi-normed (normed) if for every $u \in \mathcal{U}(R)$ there exists $v \in \mathcal{U}(R)$ such that $\|v\| \leq\|u\|$ and $\left\|v^{-1}\right\| \leq\|u\|^{-1}$.

Observe that absolutely semi-normed rings are almost absolutely semi-normed. Trivial examples of absolutely semi-normed rings are the absolutely semi-valued rings and the algebras of continuous functions in view of Example 3. On the other hand, almost absolutely semi-normed rings contain absolutely invertibles in view of Lemma 1(6), so in particular they must be unital $(\|1\|=1)$ in virtue of Lemma 1(5).

Example 4 Consider the commutative ring $\mathbb{Z}_{4}:=\frac{\mathbb{Z}}{4 \mathbb{Z}}$. We can define a ring norm on $\mathbb{Z}_{4}$ as follows:

$$
\|\overline{0}\|=0,\|\overline{1}\|=a,\|\overline{2}\|=\|-\overline{2}\|=b,\|\overline{3}\|=\|-\overline{1}\|=\|\overline{1}\|=a .
$$

Notice that it is just necessary and sufficient that $a \geq 1$ and $0<b \leq 2 a$ for $\|\cdot\|$ to be a ring norm. Observe that $\mathbb{Z}_{4}$ becomes a discrete topological space endowed with the previous norm and thus it is trivially a complete metric space. Finally,

- $\mathcal{U}\left(\mathbb{Z}_{4}\right)=\{\overline{1}, \overline{3}\}, \operatorname{t\ell d}(0)=\ell d(0)=\operatorname{rd}(0)=\operatorname{trd}(0)=\{\overline{0}, \overline{2}\}$, bd $\left(\mathcal{U}\left(\mathbb{Z}_{4}\right)\right)=\varnothing$.

- If $a=1$, then $\|\overline{1}\|=1=\left\|(\overline{1})^{-1}\right\|^{-1}$ and $\|\overline{3}\|=1=\left\|(\overline{3})^{-1}\right\|^{-1}$, so $\mathcal{U}_{1}\left(\mathbb{Z}_{4}\right)=\{\overline{1}, \overline{3}\}=$ $\mathcal{U}\left(\mathbb{Z}_{4}\right)$ and $\mathbb{Z}_{4}$ is absolutely normed.

- $\|\overline{2} \cdot \overline{2}\|=\|\overline{4}\|=\|\overline{0}\|=0<b^{2}=\|\overline{2}\|\|\overline{2}\|$, so $\mathbb{Z}_{4}$ is not absolutely valued.

Let us prove now that the closure of the invertibles in complete almost absolutely seminormed rings is contained in the set of bilateral topological divisors of zero. We first need the following technical lemma.

Lemma 2 Let $R$ be a complete normed ring. Let $\left(u_{n}\right)_{n \in \mathbb{N}} \subseteq \mathcal{U}(R)$ be convergent to some $r \in R$. The following conditions are equivalent:

1. $r \notin \mathcal{U}(R)$.

2. Every subsequence of $\left(u_{n}^{-1}\right)_{n \in \mathbb{N}}$ is unbounded.

3. $\left(u_{n}^{-1}\right)_{n \in \mathbb{N}}$ is unbounded.

Proof Suppose first that $r \notin \mathcal{U}(R)$ and assume to the contrary that there exists a bounded subsequence $\left(u_{n_{k}}^{-1}\right)_{k \in \mathbb{N}}$ with bound $M>0$. Notice then that

$$
\left\|u_{n_{i}}^{-1}-u_{n_{j}}^{-1}\right\|=\left\|u_{n_{i}}^{-1}\left(u_{n_{j}}-u_{n_{i}}\right) u_{n_{j}}^{-1}\right\| \leq\left\|u_{n_{i}}^{-1}\right\|\left\|u_{n_{j}}-u_{n_{i}}\right\|\left\|u_{n_{j}}^{-1}\right\| \leq 2 M\left\|u_{n_{j}}-u_{n_{i}}\right\|
$$

for every $i, j \in \mathbb{N}$. This implies that $\left(u_{n_{k}}^{-1}\right)_{k \in \mathbb{N}}$ is a Cauchy sequence and thus convergent to some $s \in R$. In this case, it is trivial that $r s=s r=1$, reaching the contradiction that $r \in \mathcal{U}(R)$. Finally, assume that $\left(u_{n}^{-1}\right)_{n \in \mathbb{N}}$ is unbounded and let us suppose to the contrary that $r \in \mathcal{U}(R)$. The continuity of the inversion map (see Theorem 8) forces that $\left(u_{n}^{-1}\right)_{n \in \mathbb{N}}$ converges to $r^{-1}$. This contradicts the fact that $\left(u_{n}^{-1}\right)_{n \in \mathbb{N}}$ is unbounded. 
We now obtain the following theorem from Lemma 2, which extends [9, Proposition 1.2.28] to a larger class of normed rings than just the Banach algebras.

Theorem 11 If $R$ is a complete almost absolutely normed ring, then $\mathrm{bd}(\mathcal{U}(R)) \subseteq t \ell d(0) \cap$ $\operatorname{trd}(0)$.

Proof Let $\left(u_{n}\right)_{n \in \mathbb{N}} \subseteq \mathcal{U}(R)$ be convergent to some $r \in R \backslash \mathcal{U}(R)$. According to Lemma 2, there exists a subsequence $\left(u_{n_{k}}\right)_{k \in \mathbb{N}}$ such that $\left\|u_{n_{k}}^{-1}\right\| \rightarrow \infty$ as $k \rightarrow \infty$. By hypothesis, for every $k \in \mathbb{N}$, there exists $s_{k} \in \mathcal{U}(R)$ such that $\left\|s_{k}\right\| \leq\left\|u_{n_{k}}^{-1}\right\|$ and $\left\|s_{k}^{-1}\right\| \leq\left\|u_{n_{k}}^{-1}\right\|^{-1}$. Thus,

$$
1 \leq\left\|u_{n_{k}}^{-1}\right\|\left\|s_{k}\right\|^{-1} \leq\left\|u_{n_{k}}^{-1} s_{k}^{-1}\right\| \leq\left\|u_{n_{k}}^{-1}\right\|\left\|s_{k}^{-1}\right\| \leq 1
$$

for all $k \in \mathbb{N}$. Finally,

$$
\begin{aligned}
\left\|r\left(u_{n_{k}}^{-1} s_{k}^{-1}\right)\right\| & =\left\|s_{k}^{-1}+\left(r-u_{n_{k}}\right) u_{n_{k}}^{-1} s_{k}^{-1}\right\| \\
& \leq\left\|s_{k}^{-1}\right\|+\left\|r-u_{n_{k}}\right\|\left\|u_{n_{k}}^{-1}\right\|\left\|s_{k}^{-1}\right\| \\
& \leq\left\|u_{n_{k}}^{-1}\right\|^{-1}+\left\|r-u_{n_{k}}\right\| \rightarrow 0
\end{aligned}
$$

as $k \rightarrow \infty$. This shows that $r \in t \ell d(0)$ and hence $\operatorname{bd}(\mathcal{U}(R)) \subseteq t \ell d(0)$. In a similar way it can be shown that bd( $((R)) \subseteq \operatorname{trd}(0)$.

Since absolutely valued rings are integral domains and also free of nonzero topological divisors of zero, we trivially obtain the following corollary.

Corollary 1 Let $R$ be a complete absolutely valued ring. Then bd(U(R)) is either empty or $\{0\}$.

It is very easy to find examples of complete absolutely valued rings for which bd $(\mathcal{U}(R))=$ $\{0\}(\mathbb{R}$ or $\mathbb{C})$ and for which bd$(\mathcal{U}(R))=\varnothing(\mathbb{Z})$. Example 4 shows the existence of complete normed rings satisfying that bd(U(R)) is empty but containing topological divisors of zero, which are not approximated by invertibles.

We now provide a sufficient condition for a normed ring $R$ to satisfy bd $(\mathcal{U}(R))=t \ell d(0)=$ $\operatorname{trd}(0)$.

Corollary 2 Let $R$ be a complete almost absolutely normed ring. If $U(R)$ is dense in $R$, then $\operatorname{bd}(\mathcal{U}(R))=t \ell d(0)=\operatorname{trd}(0)$.

Proof Since $\mathcal{U}(R)$ is open and dense, we have that $\{\mathcal{U}(R)$, bd(U(R)) $\}$ is a partition of $R$. On the other hand, we know that $t \ell d(0) \cup \operatorname{trd}(0) \subseteq R \backslash \mathcal{U}(R)=\operatorname{bd}(\mathcal{U}(R)) \subseteq \operatorname{t\ell d}(0) \cap \operatorname{trd}(0)$.

Theorem 12 Let $R$ be a complete normed ring. Then either $\operatorname{cl}\left(\mathcal{U}_{1}(R)\right) \backslash U(R)=\varnothing$ or $\operatorname{cl}\left(\mathcal{U}_{1}(R)\right) \backslash \mathcal{U}(R)=\{0\}$.

Proof Suppose that there exists a sequence $\left(u_{n}\right)_{n \in \mathbb{N}} \subseteq \mathcal{U}_{1}(R)$ converging to some $r \in$ $R \backslash \mathcal{U}(R)$ with $\|r\|>0$. Since $\left(\left\|u_{n}\right\|\right)_{n \in \mathbb{N}}$ converges to $\|r\|$ and $\left\|u_{n}^{-1}\right\|=\left\|u_{n}\right\|^{-1}$ for all $n \in \mathbb{N}$, we deduce that $\left(\left\|u_{n}^{-1}\right\|\right)_{n \in \mathbb{N}}$ converges to $\|r\|^{-1}<\infty$, which implies that $\left(\left\|u_{n}^{-1}\right\|\right)_{n \in \mathbb{N}}$ is bounded. This contradicts Lemma 2. As a consequence, $\|r\|=0$, so $r=0$. 
Acknowledgements The authors would like to thank the reviewers for their valuable comments and remarks which have contributed to improve the presentation and quality of the manuscript. The first author has been supported by Research Grant PGC-101514-B-I00 awarded by the Ministry of Science, Innovation and Universities of Spain, and by the 2014-2020 ERDF Operational Programme and by the Department of Economy, Knowledge, Business and University of the Regional Government of Andalusia with Project reference: FEDER-UCA18-105867 and Ministerio de Educación y Ciencia (Grant number MTM2016- 75963-P). The second author has been supported by Project PGC2018-094431-B-100 (MICINN. Spain) and Project 8059/2019 (Universitat Jaume I). The third author is supported by MCIN/AEI/10.13039/501100011033, Project PID2019-105011GB-I00, and by Generalitat Valenciana, Project PROMETEU/2021/070.

\section{Declarations}

Conflict of interest The authors declare that they have no conflict of interest.

\section{References}

1. Dales, H.G., Feinstein, J.F.: Banach function algebras with dense invertible groups. Proc. Am. Math. Soc. 136(4), 1295-1304 (2008)

2. Kulkarni, S.H.: The group of invertible elements of a real Banach algebra. Houston J. Math. 40(3), 833-836 (2014)

3. Robertson, G.: On the density of the invertible group in $C^{*}$-algebras. Proc. Edinb. Math. Soc. 20(2), 153-157 (1976)

4. Bhatt, S.J., Dedania, H.V.: Banach algebras in which every element is a topological zero divisor. Proc. Am. Math. Soc. 123(3), 735-737 (1995)

5. Marcos, J.C., Palacios, A.R., Velasco, M.V.: A note on topological divisors of zero and division algebras. Rev. R. Acad. Cienc. Exactas Fís. Nat. Ser. A Mat. RACSAM 109(1), 93-100 (2015)

6. Żelazko, W.: On generalized topological divisors of zero. Stud. Math. 85(2), 137-148 (1987)

7. García, M.C., Rodríguez Palacios, A.: Non-associative normed algebras. Vol. 1. The Vidav-Palmer and Gelfand-Naimark theorems. In: Encyclopedia of Mathematics and its Applications, vol. 154. Cambridge University Press, Cambridge (2014)

8. Müller, V.: Spectral theory of linear operators and spectral systems in Banach algebras. In: Operator Theory: Advances and Applications, vol. 139. Birkhäuser Verlag AG, Basel (2007)

9. Roch, S., Santos, P.A., Silbermann, B.: Non-commutative Gelfand Theories: A Tool-kit for Operator Theorists and Numerical Analysts. Springer, London (2011)

10. Dummit, D.S., Foote, R.M.: Abstract algebra, 3rd edn. Wiley, Hoboken (2004)

11. Edwards, R.E.: Functional Analysis: Theory and Applications. Dover Publications Inc, New York (1995).. (Corrected reprint of the 1965 original)

12. Gamelin, T.W.: Uniform algebras, 2nd edn. Chelsea Publishing Company, New York (1984)

13. Harte, R.: Invertibility and Singularity for Bounded Linear Operators: Monographs and Textbooks in Pure and Applied Mathematics, vol. 109. Marcel Dekker Inc, New York (1988)

14. Kato, T.: Perturbation Theory for Linear Operators: Classics in Mathematics. Springer, Berlin (1995).. (Reprint of the 1980 edition)

15. Mathieu, M.: Spectral isometries. In: Topological Algebras, Their Applications, and Related Topics, vol. 67, pp. 265-269. Banach Center Publications, Warsaw (2005)

16. Waelbroeck, L.: Topological Vector Spaces and A: Lecture Notes in Mathematics, vol. 230. Springer, Berlin (1971)

17. Warner, S.: Topological Fields. Elsevier Science Publishers B.V., Amsterdam (1989)

18. Warner, S.: Topological rings: North-Holland Mathematics Studies, vol. 178. North-Holland Publishing Co., Amsterdam (1993)

19. García-Pacheco, F.J., Sáez-Martínez, S.: Normalizing rings. Banach J. Math. Anal. 14(3), 1143-1176 (2020)

20. Berberian, S.K., Halmos, P.R.: Lectures in Functional Analysis and Operator Theory. Springer, New York (1974)

21. Megginson, R.E.: An introduction to Banach space theory. In: Graduate Texts in Mathematics, vol. 183. Springer, New York (1998)

22. García-Pacheco, F.J.: Regularity in topological modules. Mathematics 8(9), 1580 (2020) 
23. García-Pacheco, F.J., Piniella, P.: Unit neighborhoods in topological rings. Banach J. Math. Anal. 9(4), 234-242 (2015)

24. García-Pacheco, F.J., Piniella, P.: Linear topologies and sequential compactness in topological modules. Quaest. Math. 40(7), 897-908 (2017)

25. García-Pacheco, F.J., Piniella, P.: Geometry of balanced and absorbing subsets of topological modules. J. Algebra Appl. 18(6), 13 (2019)

Publisher's Note Springer Nature remains neutral with regard to jurisdictional claims in published maps and institutional affiliations. 